\title{
The Relation Between Prices of Factors and Goods in General Equilibrium
}

\author{
TARADAS BANDYOPADHYAY \\ Department of Economics, University of California Riverside
}

TAPAN BISWAS

Department of Economics, University of Hull, United Kingdom

\begin{abstract}
In an $\mathrm{n} \times \mathrm{n}$ economy, the relation between commodity prices and factor prices has been presented in terms of finite variations. Using a generalization of the dominant diagonal condition on the Jacobian of the set of unit cost functions, this paper shows that a rise in the price of any commodity will bring about an increase in the earnings of the corresponding factor, making no other factor better off than that factor while the earnings of at least one other factor will not increase. Strengthening the requirement further shows that the earnings of at least one factor will decline.
\end{abstract}

JEL Classification: D0; D5; F0

\section{Introduction}

The purpose of this paper is to study the relationship between commodity prices and factor prices in terms of finite variations in a general equilibrium framework. The relationship in terms of infinitesimally small variations has been studied extensively over fifty years. The reason for this alteration, to quote Samuelson (1947) from his classic Foundations of Economic Analysis, is that "in the world of real phenomena all changes are necessarily finite, and instantaneous rates of change remain only limiting abstractions...It is imperative, therefore, that we develop the implications of our analysis for finite changes" (p. 46) ${ }^{1}$. It is well known in mathematics that stating theorems in terms of infinitesimally small variations may sometimes be misleading. For example, consider the function $y=z^{3}$ in the neighborhood of 0 . The first order derivative is 0 but it is clear that any finite change in $x$ around 0 will increase the value of $y$, i.e., $y$ is an increasing function in $x$. Any finite increase in $x$ will increase $y$ and vice versa.

Stolper and Samuelson (1941) considered a competitive economy which produces two commodities with two factors of production using linearly homogeneous and concave production technologies. They showed that an autonomous increase in the price of a commodity increases the real reward of one factor and to a decline in the real reward of the other. Regarding the importance of this result, Jones (1991) wrote: "Like the

${ }^{1}$ In economics, Morishima (1964) is one of the very first persons who argued for global comparative static analysis. He proved the Hicksian Laws and several related comparative static results for finite variations.

Originally published in Journal of Quantitative Economics (2000), 16 (2): 53-64 
proverbial Helen of Troy, this article must have launched close to a thousand subsequent articles applying the theorem, qualifying it, extending it, and providing empirical estimates.” Kuhn (1967), Chipman (1969), Kemp and Wegge (1969), Inada (1971) and Uekawa (1971), at the same time, independently felt that this result would be even more useful if it could be extended to the case of $n$ commodities and $n$ factors of production. In the original setting, the effect of an increase in the price of a commodity (say $i$ ) on factor earnings depends on the intensity of that factor in the production of commodity $i$. However, generally, in an nxn competitive economy it is not possible to measure factor intensities unambiguously. In such cases, the Stolper-Samuelson theorem is usually extended in a restricted framework which requires a unique ranking of factors for a given ranking of output. For an $n \times \mathrm{x} n$ economy, Chipman (1969) defined the Stolper-Samuelson criteria, dubbed in the literature as the weak Stolper-Samuelson condition, in which given any initial equilibrium, there exists a one to one association between commodities and factors such that a rise in the price of any commodity will bring about a more than proportionate increase in the price of the corresponding factor. Such a corresponding factor is defined as the intensive factor. A slightly stronger criterion, known as the strong Stolper-Samuelson condition, states that a rise in the price of a commodity will bring about a more than proportionate increase in the price of the corresponding intensive factor and a fall in all the remaining factor prices. The Jacobian matrix A of the set of unit cost functions satisfy the strong Stolper-Samuelson criteria if (i) A is non-negative; (ii) A is nonsingular; and (iii) $\mathrm{A}^{-1}$ has negative off-diagonal elements. ${ }^{2}$ It has been shown by Chipman that if A satisfies the weak Stolper-Samuelson criteria then A is a matrix having positive principle minors (P-matrix). Although all matrices with positive dominant diagonals are P-matrices, the converse is not true. ${ }^{3}$

In this paper, we first propose a condition, in terms of finite variations, under which commodities and factors are associated in such a way that a rise in the price of any commodity will bring about an increase in the price of the corresponding factor, making no other factor better off than that factor while price of at least one factor will fall. ${ }^{4}$ Utilizing a generalized dominant diagonal property, we introduce a monotonicity

\footnotetext{
${ }^{2}$ Chipman (1969; p.404) has given an example of a positive dominant diagonal matrix whose inverse does not have all off diagonal elements as negative numbers and does not satisfy his strong Stolper-Samuelson theorem. However, the reader will notice that, in his example, a rise in the price of any commodity i will bring about a fall in the price of at least one distinct factor $j$, as required in our theorem. We shall establish that for his strong version, one needs something more than the quasi-dominant diagonal condition.

${ }^{3}$ For the relation between various dominant diagonal matrices (e.g. generalized dominant diagonal matrix, quasi-dominant diagonal matrix), the $P$ matrix, and the Minkowski matrix etc. see Uekawa, Kemp and Wegge (1974).

${ }^{4}$ The Stolper-Samuelson property emphasizes the effect of an increase in the price of a commodity on the real reward (i.e., more than proportionate increase in price) of the corresponding (intensive) factor. Notice that our emphasis is on factor price, not on real reward.
}

Originally published in Journal of Quantitative Economics (2000), 16 (2): 53-64 


\section{BANDYOPADHYAY AND BISWAS Prices of Factors and Goods}

condition which turns out to be sufficient for such a relationship between commodity prices and factor prices to hold for finite variations. ${ }^{5}$

The importance of our analysis lies in the fact that we are concerned with finite variations. That is, we are concerned with the global version of Stolper-Samuelson type property rather than the local versions discussed in the current literature. ${ }^{6}$ Another advantage of dealing with finite differences is that we do not require the unit cost functions to be differentiable. Moreover, as a minor point, the unit cost functions are concave and continuous but the Jacobian may not exist on a set of measure zero. This does not create any problem with our approach. Finally, in the existing literature, the Jacobian of the unit cost functions is not allowed to vanish on the domain of factor prices. Consider the unit iso-cost curve associated with two products, drawn on the factor-price space $\left(\omega_{1}, \omega_{2}\right)$. If the two sets of unit iso-cost curves are tangential along a ray through the origin (as at point $a$ in figure 1), the Jacobian of the mapping vanishes and the factor-price equalization theorem does not hold. This is related to the factor intensity reversal condition. However, it is possible that two sets of unit iso-cost curves will intersect each other along a ray through the origin and at the point of intersection the curves have the same slope (with different second order derivatives) as at point $d$ in figure 1. The Jacobian of our mapping vanishes at $d$, but the Stolper-Samuelson type comparative static result holds. To understand the significance of this case, compare point $a$ with point $d$ in figure 1. These refer to two different cases of vanishing Jacobians. In the first case, $b$ and $c$ are two different points on the $\left(\omega_{1}, \omega_{2}\right)$ space but they are associated with same unit costs (prices under competition) of two commodities. By comparing points $b$ and $c$ with $a$ in figure 1, it is clear that a fall in the price of the second commodity (labelled 3 in this case) may be associated either with a fall in $\omega_{2}$ (accompanied by a rise in $\omega_{1}$ ) or with a rise in $\omega_{2}$ (accompanied by a fall in $\omega_{1}$ ). Hence, the Stolper-Samuelson type property does not hold. In the second case, compare point $d$ with point $e$. An increase in the price of the second commodity increases $\omega_{2}$ and reduces $\omega_{1}$. The direction of changes is unique and the Stolper-Samuelson type comparative static result holds.

The paper is organized as follows. The next section introduces notation and definitions. In Section 3 we present the comparative static results, related to the StolperSamuelson property. The proofs are in Section 4.

\footnotetext{
${ }^{5}$ The link between the dominant diagonal condition and the Stolper-Samuelson property was investigated in the framework of the matrix of factor shares by Mitra-Jones (1992) and Jones, Marjit, Mitra (1993).

${ }^{6}$ Although there is considerable literature on the generalization and extension of the StolperSamuelson theorem one may find major works, among others, in Chipman (1969), Kemp and Wegge (1969), Uekawa (1971), Inada (1971), Wegge and Kemp (1971), Uekawa, Wegge and Kemp (1974), Willoughby (1977), Jones and Marjit (1985), Jones and Mitra (1992), Jones, Marjit and Mitra (1993). The related interesting results are in Arrow and Hahn (1971, Theorem 10, p.258), Chang, Ethier and Kemp (1980).
}

Originally published in Journal of Quantitative Economics (2000), 16 (2): 53-64 
Figure 1.

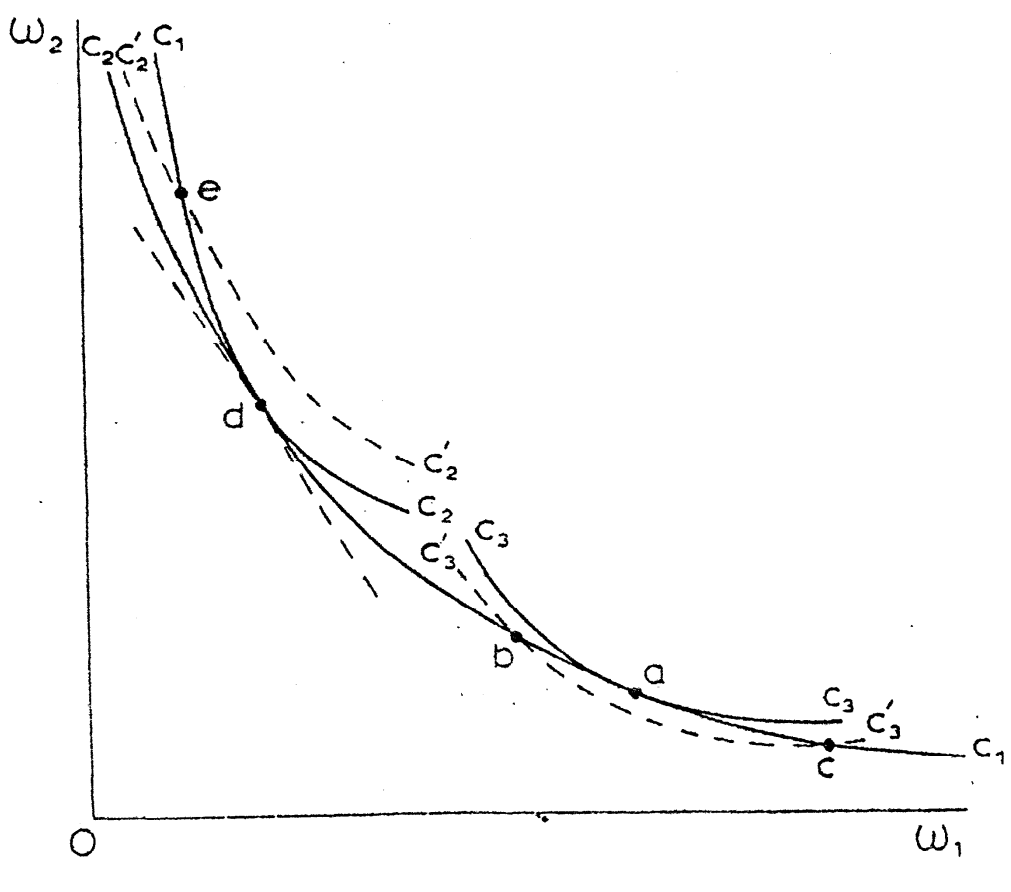

\section{Preliminaries}

The usual setting in which the Stolper-Samuelson theorem is discussed involves the production of $n$ commodities in competitive markets, each produced by the use of $n$ distinct factors of production. First, we assume that the production functions are concave and homogenous of the first degree. We also assume that all $\mathrm{n}$ commodities are produced in positive amounts. The minimum unit costs are equal to the respective prices, which are assumed to be positive. Let $p=\left(p_{1}, \ldots, p_{\mathrm{n}}\right)$ be a vector of commodity prices and let $\omega=$ $\left(\omega_{1}, \ldots, \omega_{\mathrm{n}}\right)$ be a vector of factor-prices. Given a vector of factor prices, producers select those input coefficients of a product which minimize its unit cost. Thus the input coefficients of a product are homogeneous functions of degree zero in the factor prices $\omega$. Under competition, a unit cost function can be written as, $p_{i}=f_{i}(\omega)$, which is homogeneous of degree one. Then a set of unit cost functions,

$$
P_{1}=f_{i}(\omega), \quad i=1, \ldots, n
$$

can be defined on $\Omega$ non-negative orthant of the $n$ dimensional real space $R^{n}$. In short (1) can be written as, $p=f(\omega)$, where $p, \omega \in \Omega$. Throughout this paper the function $f_{i}$ is assumed to be non-decreasing with respect to $w_{j}$, i.e., $f_{i}\left(\omega^{\prime}, \ldots, \omega_{n}^{\prime}\right) \geq f_{i}\left(\omega_{1}, \ldots, \omega_{n}\right)$ for $\omega_{j}^{\prime}>\omega_{j}$ and $\omega_{i}^{\prime}=\omega_{i}$ for all $i \neq j$. The function is said to be increasing if the weak inequality of $f_{i}$ is replaced by the strict inequality.

Originally published in Journal of Quantitative Economics (2000), 16 (2): 53-64 


\section{BANDYOPADHYAY AND BISWAS Prices of Factors and Goods}

The literature on the generalization of the Stolper-Samuelson theorem rests on various restrictions, directly or indirectly, on the Jacobian of the unit cost functions (e.g., Chipman (1969), Kemp and Wegge (1969), Uekawa (1971), Wegge and Kemp (1971), Etheir (1974), Uekawa, Wegge and Kemp (1974), Jones and Scheinkman (1977), Willoughby (1977), Jones and Mitra (1992)). For a set of increasing functions, the positive dominant diagonal property of the Jacobian implies that a simultaneous increase in the $i$ th variable and a decrease in all other variables by the same amount increases the value of the $i$ th function. To capture the essence of the argument, rewrite (1) as

$$
p_{i}=f_{i}\left(\alpha_{1 i}-\alpha_{i i}, \ldots, \alpha_{i i}, \ldots, \alpha_{n i}-\alpha_{i i}\right) \text {, }
$$

where $\alpha_{j i}=\omega_{j}+\omega_{i}$ for $j \neq i, \alpha_{i i}=\omega_{i}$ and $\alpha=\left(\alpha_{j i}\right) \in \Omega$. Note that the effect of a change in $\alpha_{j i}$ on $f_{i}$ where $i \neq j$, is the same as the effect of a change in $w_{j}$; however, any change in $\alpha_{i i}$ alone affects all other $\left(\alpha_{j i}-\alpha_{i i}\right)$ 's of the $f_{i}$ function. ${ }^{7}$

A unit cost function $f_{i}$ is said to be weakly dominant when a simultaneous increase in $\omega_{i}$ and decrease in all other $\omega_{j}^{\prime}$ s by the same amount does not decrease the value of $f_{i}$. A unit cost function $f_{i}$ is said to be dominant when a simultaneous increase in $\omega_{i}$ and decrease in all other $\omega_{j}$ 's by the same amount increases the value of $f_{i}$.

A set of unit cost functions $f_{i}(i=1, \ldots, n)$ satisfies weakly positive dominance condition (WPD) if every $f_{i}$ is weakly dominant and there is a sequence $i(0), i(1), \ldots, i(k)$, where $i(0)$ $=i$, such that for $s=0,1, \ldots, k-1, f_{i(s)}$ is increasing; in $\omega_{i(s+1)}$, and $\mathrm{f}_{i(k)}$ is dominant. $^{8}$

In words, WPD requires that the goods and the factors may be ranked in such a way that the price of good $i$ is either increasing in the price of factor $i$, or if the increase in the price of factor $i$ and an equal reduction in all other factor-prices does not reduce the price of commodity $i$, then there exists a sequence $i(0), i(1), i(2),. \ldots, i(k)$ with $i(0)=i$ such that the price of commodity $i(\mathrm{~s})$ is increasing in the price of factor $i(s+1)$ for $\mathrm{s}=0,1, \ldots, k-1$ and $f_{i(k)}$ is dominant (i.e., in equation (1) $)^{i}$ the price of commodity $i(k)$ is increasing in $\left.\alpha_{i(k) i(k)}\right)$. In a two-good and two-factor world, WPD is trivially satisfied when the Jacobian of the unit cost function does not alter sign. For differentiable unit cost functions, the positive quasi-dominant diagonal property of the Jacobian implies WPD. To put it differently, the WPD property is similar to a "dominant diagonal" type condition on the Jacobian of the set of (differentiable) unit cost functions.

\footnotetext{
${ }^{7}$ By introducing a transformation function, the relation (1)' can be written as $p_{i}=F_{i}\left(\alpha_{1 i} \ldots\right.$, $\left.\alpha_{i i}, \ldots . . \alpha_{n i}\right)$. Notice that the effects of a change in $\omega_{j}$ on $F_{i}$ and $f_{j}$, where $i \neq j$, are same; however, any change in $\omega_{1}$ alone affects all other arguments of the $F_{i}$ function. Thus to examine the effect of an increase in only the ith argument of $F_{i}$ one must simultaneously increase in $\omega_{i}$ and decrease in all other $\omega_{j}^{\prime}$ s by the same amount.

${ }^{8}$ See footnote 7. Alternatively, with $F_{i}$ defined for $i=1, \ldots, n$, a set of unit cost functions $f_{i}(i=$ $1, \ldots, n)$ is said to be $W P D$ if every $F_{i}$ is non-decreasing in $\alpha_{i i}$ and there is a sequence $i(0)$, $i(1), \ldots, i(k)$, where $i(0)=i$, such that for $s=0,1, \ldots, k-1, F_{i(\mathrm{~s})}$ is increasing in $\alpha_{i(\mathrm{~s}) i(\mathrm{~s}+1)}$ and $F_{i(\mathrm{k})}$ is increasing in $\alpha_{i(\mathrm{k}) i(\mathrm{k})}$.
}

Originally published in Journal of Quantitative Economics (2000), 16 (2): 53-64 
In an earlier work (1994), we presented a mapping which satisfies WPD but its Jacobian is neither a $P$-matrix nor a positive dominant diagonal matrix. The following example shows that a mapping, whose Jacobian is a positive $P$-matrix (a matrix which is positive as well as a $P$-matrix), does not necessarily satisfy WPD.

$$
A=\left[\begin{array}{lll}
5 & 2 & 4 \\
1 & 2 & 1 \\
1 & 2 & 1
\end{array}\right] \quad A^{-1}=(1 / 11)\left[\begin{array}{ccc}
5 & -2 & -6 \\
-1 & 7 & -1 \\
-3 & -1 & 8
\end{array}\right]
$$

Utilizing the WPD condition we shall present a comparative static result similar to the weak Stolper-Samuelson property in the following section. Now, to establish a result which is parallel to a stronger version of Stolper-Samuelson relation, we introduce the condition of irreducibility. A set of unit cost functions $p=f(\omega)$, where $p, \omega \in \Omega$ is said to be irreducible if there exists some $i, j$ such that $p_{i}$ is an increasing function of $\omega_{i}$ for $i \in j$ and $j \notin J$ for any non-empty subset $J$ of indices.

\section{The Relation Between Commodity Prices And Factor Earnings In A Many-Commodity, Many-Factor World}

\section{THEOREM.}

Let a set of unit cost functions f satisfy the WPD condition. (1) If $\delta \mathrm{p}_{\mathrm{k}}>0$ and $\delta \mathrm{p}_{\mathrm{i}}=0$ for all $i \neq k$, then $/ \delta \omega_{i} / \leq \delta \omega_{k}>0$ and $\delta \omega_{i} \leq 0$ for some i. (2). If a set of unit cost functions forms an irreducible structure then if

$\delta p_{k}>0$ and $\delta p_{i}=0$ for all $i \neq k$, then $/ \delta \omega_{i} / \leq \delta \omega_{k}>0$ and $\delta \omega_{i}<0$ for some i.

In words, the first part of the theorem says that for a set of unit cost functions that satisfies WPD, any finite increase in the price of any one good, say commodity $k$, will increase the earnings of the k-th factor, making no other factor better off than the k-th factor while earnings of at least one other factor will not increase. The second part says that for a set of irreducible unit cost functions that satisfy WPD, any finite increase in the price of any one good, say commodity $k$, the earnings of the k-th factor will increase, making no other factor better off than the k-th factor while earnings of at least one other factor will decrease. Clearly, the first part of the theorem is akin to the weak Stolper-Samuelson condition, while the second part is related to, though much weaker than, the strong Stolper-Samuelson condition.

$R E M A R K 1$, If there exists transformed variables $q_{i}$ such that $q_{i}=\phi_{i}\left(\omega_{i}\right)$, where $\phi_{i}$ is increasing in $\omega_{i}$; and $p=f\left(\phi^{-1}(q)\right)=\Theta(q)$ satisfies the WPD condition, then our result also holds because $\delta \omega_{i}$ and $\delta q_{i}$ vary in the same direction. Note that in the case of differentiable mappings, this underlines the relationship between the quasi-dominant diagonal property of the Jacobians and the Stolper-Samuelson property in terms of nominal factor rewards. The weights for quasi-dominance are generated by $d_{i}=\left(\phi_{i}^{\prime}\right)^{-1}$.

$R E M A R K 2$. In figure 1 , at $d$, the Jacobian matrix of the mapping $f$ is singular. However, any given $\left(p_{1}, p_{2}\right)$ is associated with a unique $\left(\omega_{1}, \omega_{2}\right)$. In this case, if we construct the 


\section{BANDYOPADHYAY AND BISWAS Prices of Factors and Goods}

matrix relationship described by equation (4) in Section 4, we must have a nonsingular $\left[a_{i j}\right]$ matrix since $\delta \omega$ must be a null vector. Thus the singularity of the Jacobian does not necessarily imply the singularity of the $\left[a_{i j}\right]$ matrix associated with finite differences. Here we have required a particular sign pattern of the $\left[a_{i j}\right]$ matrix or, as REMARK 1 suggests, of the matrix $[d]\left[a_{i j}\right]$, where $d$ is a diagonal matrix with $d_{j}>0$.

It is obvious that if the mapping $f$ is differentiable everywhere and if its Jacobian is a nonnegative matrix which has the property of having a positive dominant diagonal, then $f$ must satisfy the WPD condition. Denote $W_{i} \equiv\left(\omega_{i} / p_{n}\right)$ and $P_{i} \equiv\left(p_{i} / p_{n}\right)$. Chipman's strong Stolper-Samuelson property states that, for any arbitrarily chosen numeraire $\mathrm{P}_{\mathrm{n}}$, if $\delta p_{n}>0$, then $\delta W_{a}>0$ and $\delta W_{i}<0$ for all $i \neq n$. Chipman considered the mapping, $f_{c}: \log W \rightarrow \log P$, whose Jacobian is a stochastic matrix, that is, off-diagonal elements are non-negative and row sum equals to unity. In terms of the Jacobian of the mapping $f_{c}$, say matrix A, the strong Stolper-Samuelson theorem requires that the diagonal elements of $A^{-1}$ to be greater than unity. Given the example below, he showed that the matrix $\mathrm{A}$ having a positive dominant diagonal is not sufficient for the strong Stolper-Samuelson theorem because the off-diagonal elements of $A^{-1}$ are not all negative. ${ }^{9}$ However, the weak Stolper-Samuelson theorem in real rewards is satisfied because all the diagonal elements are greater than unity. ${ }^{10}$

$$
A=\left[\begin{array}{ccc}
8.2 & 0.2 & 0 \\
0.2 & 0.6 & 0.2 \\
0 & 0.2 & 0.8
\end{array}\right] \quad \mathrm{A}^{-1}=\left[\begin{array}{ccc}
11 / 8 & -1 / 2 & 1 / 8 \\
-1 / 2 & 2 & -1 / 2 \\
1 / 8 & -1 / 2 & 11 / 8
\end{array}\right]
$$

Furthermore, the example above; suggests that an increase in $p_{1}$ causes $w_{1}$ to rise and $w_{2}$ to fall. An increase in $p_{2}$ raises $w_{2}$ and reduces both $w_{1}$ and $w_{3}$. An increase in $p_{3}$ increases $w_{3}$ and reduces $w_{2}$. Therefore, the second part of our theorem is satisfied. However, Chipman requires that all other factor prices must fall.

An interesting observation emerging from the literature on the link between the dominant diagonal condition and the Stolper-Samuelson property is that, instead of the dominant diagonal property of the factor shares, it is the dominant diagonal property of the matrices of differences in factor shares or matrices of differences in ratios of factor shares which is more directly linked to the Stolper-Samuelson theorem. However, our main result establishes a relationship between a generalized dominant diagonal property (WPD) of the input coefficient matrix and a weaker property in which commodities and factors are associated in such a way that a rise in the price of any commodity will bring

\footnotetext{
${ }^{9}$ Chipman (1969) had also given an example to show that the dominant diagonal condition is not necessary for the Stolper-Samuelson theorem to hold.

${ }^{10}$ If a commodity that requires only one input, then even the weak Stolper-Samuelson theorem does not hold. For example, if $a_{31}=a_{32}=0$ and $a_{33}=1$, then $\operatorname{dlog} W_{3} / \operatorname{dlog} P_{3}=1$.
}

Originally published in Journal of Quantitative Economics (2000), 16 (2): 53-64 
about an increase in the price of the corresponding factor, not necessarily real rewards, making no other factor better off than that factor while the price of at least one will fall.

\section{Proofs}

This section contains four parts. First, we shall construct an nxn matrix which is the counterpart of the Jacobian of a set of differentiable unit cost functions. Second, we shall present a basic mathematical result. Then, utilizing this result, we shall establish the main result of the paper. Lastly, we shall prove theorem 2.

Following Duffin (1948) and Bandyopadhyay and Biswas (1994), we describe the procedure of constructing an $n \times n$ matrix in $a_{i j}$ 's corresponding to the given set of unit cost functions $p_{i}=f_{i}\left(\omega_{1}, \ldots, \omega_{n}\right), \quad i=1, \ldots, n$. For any assigned commodity price vector $p=\left(p_{1}, p_{2}, \ldots, p_{n}\right)$, if there exist two factor price vectors $\omega$ and, $\omega$ ' define

$$
\alpha_{i i}=\omega_{i}, \alpha_{j i}=\left(\omega_{j}+\omega_{i}\right) \text { for } j \neq i \text { and } \alpha_{i i}^{\prime}=\omega_{i}^{\prime}, \alpha_{j i}^{\prime}=\left(\alpha_{j}^{\prime}+\omega_{i}^{\prime}\right) \text { for } j \neq i
$$

First consider the case of $\alpha_{11} \leq \alpha_{11}^{\prime}$. By definition,

$$
\begin{aligned}
& 0=f_{1}\left(\alpha_{11}^{\prime},\left(\alpha_{21}^{\prime}-\alpha_{11}^{\prime}\right), \ldots,\left(\alpha_{n 1}^{\prime}-\alpha_{11}^{\prime}\right)\right)-f_{1}\left(\alpha_{11},\left(\alpha_{21}-\alpha_{11}\right), \ldots,\left(\alpha_{n 1}-\alpha_{11}\right)\right) \\
& =\left[f_{1}\left(\alpha_{11}^{\prime},\left(\alpha_{21}^{\prime},-\alpha_{11}^{\prime}\right), \ldots,\left(\alpha_{n 1}^{\prime},-\alpha_{11}^{\prime}\right)\right)-f_{1}\left(\alpha_{11},\left(\alpha_{21}^{\prime}-\alpha_{11}\right), \ldots,\left(\alpha_{n 1}^{\prime}-\alpha_{11}\right)\right)\right] \\
& +\left[f_{1}\left(\alpha_{11},\left(\alpha_{21}^{\prime},-\alpha_{11}\right), \ldots,\left(\alpha_{n 1}^{\prime}-\alpha_{11}\right)\right)-f_{1}\left(\alpha_{11},\left(\alpha_{21}-\alpha_{11}\right), \ldots,\left(\alpha_{n 1}^{\prime}-\alpha_{11}\right)\right)\right]+\ldots \\
& +\left[f_{1}\left(\alpha_{11},\left(\alpha_{21},-\alpha_{11}\right), \ldots,\left(\alpha_{(n-1) 1}-\alpha_{11}\right),\left(\alpha_{n 1}^{\prime}-\alpha_{11}\right)\right)-f_{1}\left(\alpha_{11},\left(\alpha_{21}-\alpha_{11}\right), \ldots,\left(\alpha_{n 1}-\alpha_{11}\right)\right)\right]
\end{aligned}
$$

Now define, for $\alpha^{\prime}{ }_{j 1} \neq \alpha_{j 1}$,

$a_{1 j}=\left[f_{1}\left(\left(\alpha_{11}, \ldots,\left(\alpha_{(j-1) 1}-\alpha_{11}\right),\left(\alpha_{j 1}^{\prime}-\alpha_{11}\right), \ldots,\left(\alpha_{n 1}^{\prime}-\alpha_{11}\right)\right)-f_{1}\left(\left(\alpha_{11}, \ldots,\left(\alpha_{j 1}-\alpha_{11}\right),\left(\alpha_{(j+1) 1}^{\prime}-\alpha_{11}\right), \ldots,\left(\alpha_{n 1}^{\prime}-\alpha_{11}\right)\right)\right] \Delta\right.\right.$ where $\Delta=\left(\alpha^{\prime}{ }_{j 1}-\alpha_{j 1}\right)^{-1}$; otherwise, for $\alpha_{1 j}^{\prime}=\alpha_{j 1}, \alpha_{1 j}=1$. Since $\alpha^{\prime}{ }_{j 1} \geq \alpha_{11}^{\prime} \geq \alpha_{11}$ for all $j$, $a_{1 j}$ 's are non-negative by WPD.

Next consider the case of $\alpha_{11} \geq \alpha_{11}^{\prime}$. Define, for $\alpha_{j 1}^{\prime} \neq \alpha_{j 1}$,

$a_{1 j}=\left[f_{1}\left(\alpha_{11}^{\prime}, \ldots,\left(\alpha_{(j-1) 1}^{\prime}-\alpha_{11}^{\prime}\right),\left(\alpha_{j 1}^{\prime}-\alpha_{11}^{\prime}\right), \ldots,\left(\alpha_{n 1}-\alpha_{11}^{\prime}\right)\right)-f_{1}\left(\alpha_{11}^{\prime}, \ldots,\left(\alpha_{j 1}^{\prime}-\alpha_{11}^{\prime}\right),\left(\alpha_{(j+1) 1}-\alpha_{11}^{\prime}\right), \ldots\right.\right.$, $\left.\left.\left(\alpha_{n 1}^{\prime}-\alpha_{11}^{\prime}\right)\right)\right] \Delta^{*}$, where $\Delta^{*}=\left(\alpha_{j 1}-\alpha_{j 1}^{\prime}\right)^{-1}$; otherwise, for $\alpha_{j 1}^{\prime}=\alpha_{j 1}, a_{1 j}=1$. Now, for this case one can easily construct the relation similar to equation (2).

For $\delta \omega=\omega^{\prime}-\omega$, we have $\alpha^{\prime}{ }_{j 1}-\alpha_{j 1}=\delta \omega_{j}+\delta \omega_{1}$ for all $j \neq 1$ and $\alpha_{11}^{\prime}-\alpha_{11}=\delta \omega_{1}$. Similarly, $a_{i j}$ 's for all $i, j=1, \ldots, n$, may be defined. Utilizing the definition of $a_{i j}$ and following the construction of (2), we obtain

$$
0=\sum_{\substack{j=1 \\ j \neq 1}}^{n} a_{i j}\left(\delta \omega_{j}+\delta \omega_{i}\right)+a_{i i} \delta \omega_{i}, i=1, \ldots, n
$$

Note that if $f$ satisfies WPD, then $a_{i} \geq 0$. This completes the construction of our $n \times n$ matrix. 
Next, in order to establish that for a mapping $f$ which satisfies WPD, if $\delta p_{k}>0$ and $\delta p_{i}=0$ for all $i \neq k$, then $\delta \omega_{k}>0,\left|\delta \omega_{i}\right| \leq \delta \omega_{k}$ and $\delta \omega_{i} \leq 0$ for some $i$, we shall use the following lemma.

LEMMA. Let $f$ satisfy WPD. If for all $i \neq h, \delta p_{i}=0$, then $\delta \omega_{n}=0$ implies $\delta \omega_{i}=0$ for all $i$.

PROOF. ${ }^{11}$ Let $\delta p_{i}=0$ for all $i \neq h$ and $\delta \omega_{h}=0$. On the contrary, suppose $\delta \omega_{i} \neq 0$ for some $i$. Without any loss of generality, assume $\delta \omega_{i}>0$ for some $i$. We shall show that there must exist some $\delta \omega_{j}$ such that $\left|\delta \omega_{j}\right|>\left|\delta \omega_{i}\right|$. Now from (3) we have,

$$
0=\sum_{j=1}^{n} a_{i j}\left(\delta \omega_{j}+\delta \omega_{i}\right)+a_{i i}^{*} \delta \omega_{i} \text {, where } a^{*}{ }_{i i}=a_{i i}+a_{i h} .
$$

First consider $a^{*}{ }_{i i}>0, a_{i i}^{*} \delta \omega_{i}>0$. Since $a_{i j} \geq 0$, by WPD, there exists $\delta \omega_{j}<0$ such that $\left|\delta \omega_{j}\right|>\left|\delta \omega_{i}\right|$. If $a_{i i}^{*}=0$, once again by WPD, there exists an element 's' in the index set such that $f_{i}$ is increasing in $\alpha_{i s}$ which implies that $a_{i s}$ is an increasing function of $\omega_{s}$. If $\delta \omega_{i} \neq-\delta \omega_{s}$, then $a_{i s}>0$. Suppose $\delta \omega_{i}<-\delta \omega_{s}$, obviously our search for a $\delta \omega_{j}$, such that $\left|\delta \omega_{j}\right|>\left|\delta \omega_{i}\right|$, ends with the choice of $j=s$. Again, if $\delta \omega_{j}>-\delta \omega_{s}$, since $a^{*}{ }_{i i} \delta \omega_{j}=0$ and $a_{i s}\left(\delta \omega_{s}+\delta \omega_{i}\right)>0$, by (4) there exists some $\delta \omega_{j}<0$ and $\left|\delta \omega_{j}\right|>\left|\delta \omega_{i}\right|$.

Suppose $a^{*}{ }_{i i}=0$ and $-\delta \omega_{n}=\delta \omega_{i}$. Consider the relation (s) of (3):

$$
0=\sum_{\substack{j=1 \\ j \neq s, h}}^{n} a_{s j}\left(\delta \omega_{j}+\delta \omega_{s}\right)+a_{s s}^{*} \delta \omega_{s} \text {, where } a_{s s}^{*}=a_{s s}+a_{s h} .
$$

By WPD either there exists a positive $\delta \omega_{j}$ such that $\left|\delta \omega_{j}\right|>\left|\delta \omega_{s}\right|=\left|\delta \omega_{i}\right|$ or there exists ' $\mathrm{t}$ ' in the index set such that $\delta \omega_{t}$ is positive and $\left|\delta \omega_{t}\right|=\left|\delta \omega_{s}\right|=\left|\delta \omega_{i}\right|$. In the former situation our search for a $\delta \omega_{j},\left|\delta \omega_{i}\right|>\left|\delta \omega_{i}\right|$ ends. Otherwise we proceed to consider the relation (t) of (3):

$$
0=\sum_{\substack{j=1 \\ j \neq t, h}}^{n} a_{t j}\left(\delta \omega_{j}+\delta \omega_{i}\right)+a^{*}{ }_{t t} \delta \omega_{t} \text {, where } a^{*}{ }_{t t}=a_{t t}+a_{t h} .
$$

Once again, by WPD, this search must end. If not, then we have to continue until we reach a situation where, $\left|\delta \omega_{k}\right|=\left|\delta \omega_{g}\right|=\ldots=\left|\delta \omega_{s}\right|=\left|\delta \omega_{i}\right|$. Clearly $a_{k k}>0$. Furthermore,

$$
0=\sum_{\substack{j=1 \\ j \neq k, h}}^{n} a_{k j}\left(\delta \omega_{j}+\delta \omega_{k}\right)+a_{k k} \delta \omega_{k} \text {, where } a_{k k}=a_{k k}+a_{k h} \text {. }
$$

\footnotetext{
${ }^{11}$ The proof is very similar to the proof of Theorem 1 in Bandyopadhyay and Biswas (1994). We repeat the proof since the similar argument would be used in establishing our result.
}

Originally published in Journal of Quantitative Economics (2000), 16 (2): 53-64 
Depending on whether $\delta \omega_{k}$ is positive or negative now there must exist a negative or positive $\delta \omega_{j}$ such that $\left|\delta \omega_{j}\right|>\left|\delta \omega_{k}\right|=\ldots=\left|\delta \omega_{i}\right|$ to satisfy this relation.

Now suppose $\delta \omega_{i} \neq 0$. Consider a $\delta \omega_{m}$ such that $\left|\delta \omega_{m}\right| \geq\left|\delta \omega_{i}\right|$ for all $i$. We have just shown above that WPD requires the existence of a $\delta \omega_{k}$ such that $\left|\delta \omega_{k}\right|>\left|\delta \omega_{m}\right|$ which contradicts the choice of $\delta \omega_{m}$. Therefore, $\delta \omega_{i}=0$. This completes the proof of the lemma. [Q.E.D.]

REMARKS 3: This lemma implies that for any given $p, \delta \omega=0$.

PROOF OF THE THEOREM (1): First we show that if $\delta p_{k}>0$ and $\delta p_{i}=0$ for all $i \neq k$, then $\delta \omega_{k}>0$. Consider Relation (3).

$$
0=\sum_{j=1}^{n} a_{i j}\left(\delta \omega_{j}+\delta \omega_{i}\right)+a_{i i} \delta \omega_{i}, \quad 1=1, \ldots, n .
$$

Without any loss of generality, let $k=n$. Suppose $\delta \omega_{n}=0$. Then,

$$
\begin{aligned}
& 0=\left(a_{11}+a_{1 n}\right) \quad \delta \omega_{1}+\ldots \ldots .+a_{1, n-1}\left(\delta \omega_{n-1}+\delta \omega_{1}\right) \\
& \cdot \\
& 0=\left(a_{n-1,1}\left(\delta \omega_{1}+\delta \omega_{n-1}\right)+\ldots . .+\left(a_{n-1, n-1}+a_{n-1, n}\right) \delta \omega_{n-1}\right.
\end{aligned}
$$

Using lemma, $\delta \omega_{1}=\ldots=\delta \omega_{n+1}=0$. Since, by assumption, $\delta \omega_{n}=0$, the relation, $0<\delta p_{n}=a_{n 1}\left(\delta \omega_{1}+\delta \omega_{n}\right)+\ldots+a_{n n} \delta \omega_{n}+$ cannot hold good. Suppose then $\delta \omega_{n}<0$. Then,

$$
0=\sum_{\substack{j=1 \\ j \neq 1}}^{n} a_{i j}\left(\delta \omega_{j}+\delta \omega_{n}\right)+a_{i i} \delta \omega_{i}, \quad i=1,2, \ldots, n-1,
$$

and

$$
0=a_{n 1}\left(\delta \omega_{1}+\delta \omega_{n}\right)+\ldots . .+\left[a_{n n}-\left(\delta p_{n} / \delta \omega_{n}\right)\right] \delta \omega_{n} .
$$

Since $\delta \omega_{n}=0,\left[a_{n n}-\left(\delta p_{n} / \delta \omega_{n}\right)\right]>0$. Again, using the arguments in the proof of the lemma, one can show that $\delta \omega_{1}=0$ for all $i$. This contradicts the assumption $\delta \omega_{n}<0$. Therefore, $\delta \omega_{n}$ must be strictly positive.

Next we show that if $\delta p_{k}>0$ and $\delta p_{i}=0$ for all $i \neq k$, then $\left|\delta \omega_{i}\right| \leq \delta \omega_{k}$. Without any loss of generality assume $k=n$. By the discussion of the previous paragraph, $\delta \omega_{n}>0$. Let $\left(\delta \omega_{i}+\delta \omega_{n}\right)<0$ for some $i$. Then, from relation (3),

$$
0=\sum_{j=1}^{n} a_{i j}\left(\delta \omega_{j}+\delta \omega_{i}\right)+a_{i i} \delta \omega_{i}, \quad i=1,2, \ldots, n-1,
$$

and

$$
0=a_{n 1}\left(\delta \omega_{1}+\delta \omega_{n}\right)+\ldots . .+\left[a_{n i}-\delta p_{n} /\left(\delta \omega_{i}+\delta \omega_{n}\right)\right]\left(\delta \omega_{i}+\delta \omega_{n}\right)+\ldots+a_{n n} \delta \omega_{n}
$$

Originally published in Journal of Quantitative Economics (2000), 16 (2): 53-64 
Since $\left(\delta \omega_{i}+\delta \omega_{n}\right)<0\left[a_{n i}-\delta p_{n} /\left(\delta \omega_{i}+\delta \omega_{n}\right)\right]>0$. Again, using the arguments in the proof of the lemma, we can prove that $\delta \omega_{1}=\ldots=\delta \omega_{n}=0$. This contradicts our assumption that $\left(\delta \omega_{1}+\delta \omega_{n}\right)<0$. Therefore,

$$
\delta \omega_{n} \geq-\delta \omega_{i} \text { for all } i \text {. }
$$

We shall complete our proof by showing, $\delta \omega_{n} \geq \delta \omega_{i}$. Suppose $\delta \omega_{n}<\delta \omega_{i}$ for some $i$. Then by (5),

$$
\delta \omega_{i}+\delta \omega_{i}>\delta \omega_{j}+\delta \omega_{n} \geq 0, \quad j=1,2, \ldots n
$$

But by definition

$$
0=\sum_{\substack{j=1 \\ j \neq 1}}^{n} a_{i j}\left(\delta \omega_{j}+\delta \omega_{i}\right)+a_{i i} \delta \omega_{i}
$$

Given WPD, this is impossible, since $\left(d \omega_{j}+\delta \omega_{i}\right)>0$ for all $j \neq i$ and $\delta \omega_{i}>0$. Therefore,

$$
\delta \omega_{n} \geq \delta \omega_{i} \text { for all } i \text {. }
$$

Utilizing equation (5) and (6) it is immediate that for $\delta p_{k}>0$ and $\delta p_{i}=0$ and $i \neq k,\left|\delta \omega_{i}\right| \leq \delta \omega_{k}$.

Now, to show that if $\delta p_{k}>0$ and $\delta p_{i}=0$ for all $i \neq k$ then $\delta \omega_{i} \leq 0$ for some $i$, consider $\delta \omega_{i}>0$ for all $i$. By WPD, for any $i \neq k$, this will violate the relation, $0=a_{i 1}\left(\delta \omega_{1}+\delta \omega_{i}\right)+\ldots+a_{i n}\left(\delta \omega_{n}+\delta \omega_{i}\right)$. This completes the proof of the first part of the theorem.

PROOF OF THE THEOREM (2): It remains to be shown that for a set of irreducible unit cost functions $f$, if $\delta p_{k}>0$ and $\delta p_{i}=0$ for all $i \neq k$, then $\delta \omega_{i}<0$ for some $i$.

Suppose the relation (1) is irreducible. Then there exist some $i=k$ such that $f_{i}$ is increasing in $\left(\delta \omega_{k}+\delta \omega_{i}\right)$. Consider,

$$
0=a_{i 1}\left(\delta \omega_{1}+\delta \omega_{i}\right)+\ldots+a_{i i} \delta \omega_{i}+\ldots+a_{i k}\left(\delta \omega_{k}+\delta \omega_{i}\right)+\ldots+a_{i n}\left(\delta \omega_{n}+\delta \omega_{i}\right) .
$$

We claim that $\delta \omega_{i}<0$. If not, then $\left(\delta \omega_{k}+\delta \omega_{i}\right)>0$. Since $a_{i k}$ is an increasing function, $a_{i k}\left(\delta \omega_{k}+\delta \omega_{i}\right)>0$ Since other terms are non-negative, the above equations cannot hold true. This violates $\delta p_{i}=0$ for $i \neq k$. [Q.E.D.]

\section{References}

Arrow, K. J., Hahn, F. H., General Competitive Analysis, San Francisco: Holden-Day (1971).

Bandyopadhyay, T. and Biswas, T., Global univalence when functions are not necessarily continuous, Journal of Mathematical Economics 23, 435-450 (1994).

Chang, W., Ethier, W., Kemp, M., The theorems on international trade with joint production, Journal of International Economics 10. 377-94 (1980).

Originally published in Journal of Quantitative Economics (2000), 16 (2): 53-64 
Chipman, J. S., Factor price equalization and the Stolper-Samuelson theorem. International Economic Review 10, 399-406 (1969).

Duffin, R. K., Non-linear networks. IIb, American Mathematical Society Bulletin 54, 119127 (1948).

Ethier, W., Some of the theorems of international trade with many goods and factors, Journal of International Economics 4, 199-206 (1974).

Inada, K., Production coefficient matrix and the Stolper-Samuelson condition, Econometrica 39, 219-240 (1971).

Jones, R.W. and Scheinkman, J.A., The relevance of two-sector model in trade theory, Journal of Political Economy 85, 909-935 (:977).

Jones, R. W., Reflection on the Stolper-Samuelson theorem, Current Issues in International Trade Theory: Festschrift in honor of Murray C. Kemp (1991).

Jones, R. W., Marjit, S., A simple production model with Stolper-Samuelson properties, International Economic Review 26, 565-567 (1985).

Jones, R. W., Marjit, S., Mitra, T., The Stolper-Samuelson theorem: links to dominant diagonals, in General Equilibrium, Growth and Trade, edited by R. Becker, M. Boldrin, R. Jones and W. Thompson, Academic Press, New York, 429-441 (1993).

Jones, R. W., Mitra, T., Factor shares and the Chipman condition, Presented in a Conference in Honor of John Chipman, University of Minnesota (1992).

Kemp, M.C., Wegge, L. L., On the relation between commodity prices and factor rewards, International Economic Review 10, 407-413 (1969).

Kemp. M.C., The pure theory of international trade and investment, Prentice-Hall, Englewood Cliffs (1969).

Kuhn, H. W., On two theorems on international trade, Economia Mathematica, Rome (1967).

Levhari, D., Paroush, J., Peleg, B., Efficiency analysis of multivariate distributions, Review of Economic Studies 42, 87-91. (1975).

McKenzie, L. W., Matrices with dominant diagonals and economic theory, in K. J. Arrow, S. Karlin, and P. Suppes, eds., Mathematical Methods in the Social Sciences, Stanford: Stanford University Press, 47-62 (1960).

Morishima, M., Equilibrium, Stability and Growth, Oxford: Clarendon Press (1964).

Samuelson, P.A., Foundations of Economic Analysis, Cambridge, MA: Harvard University Press (1947).

Samuelson, P. A., Prices of factors and goods in general equilibrium, Review of Economic Studies 21, 1-20 (1953).

Stolper, W. F., Samuelson, P. A., Protection and real wages, Review of Economic Studies 9, 58-73 (1941).

Uekawa, Y. Generalization of the Stolper-Samuelson theorem, Econometrica 37, 197-217 (1971).

Originally published in Journal of Quantitative Economics (2000), 16 (2): 53-64 


\section{BANDYOPADHYAY AND BISWAS Prices of Factors and Goods}

Uekawa, Y., Kemp, M., Wegge, L. L., P and PN matrices, Minkowski and Metzlermatrices and generalizations of the Stolper-Samuelson and Samuelson-Rybczynski theorems, Journal of International Economics 3, 53-76 (1974).

Wegge, L. L., Kemp, M., Generalization of the Stolper-Samuelson and SamuelsonRybczynski theorems in terms of conditional input-output coefficients, International Economic Review 10, 414-425 (1971).

Willoughby, R. A., The inverse M matrix problem, Linear Algebra Applications 18, 7594 (1977).

Originally published in Journal of Quantitative Economics (2000), 16 (2): 53-64 